\title{
Editorial
}

\section{Abstract Differential and Difference Equations}

\author{
G. M. N' Guérékata, ${ }^{1}$ T. Diagana, ${ }^{2}{ }^{\text {and A. Pankov }}{ }^{1}$ \\ ${ }^{1}$ Department of Mathematics, Morgan State University, Baltimore, MD 21251, USA \\ ${ }^{2}$ Department of Mathematics, Howard University, Washington, DC 20005, USA
}

Correspondence should be addressed to G. M. N’Guérékata, gaston.nguerekata@morgan.edu

Received 31 Decemeber 2010; Accepted 31 Decemeber 2010

Copyright $\odot 2010$ G. M. N'Guérékata etal. This is an open access article distributed under the Creative Commons Attribution License, which permits unrestricted use, distribution, and reproduction in any medium, provided the original work is properly cited.

This special issue of Advances in Difference Equations is devoted to highlight some recent developments in abstract differential equations, fractional differential equations, and difference equations and their applications to mathematical physics, engineering, and biology. It consists of 20 papers carefully selected through a rigorous peer review.

The first category of papers deals with the asymptotic and oscillatory behavior of solutions to various abstract differential equations and fractional differential equations. Periodic problems involving the scalar $p$-Laplacian equation on time scales, or $n$-species nonautonomous food chains with harvesting terms are studied using the Mawhin's continuation theorem. Some new oscillation criteria for the second-order quasilinear neutral delay dynamic equations and nonlinear delay dynamic equations on a time scale $T$, are established, improving some known results for oscillation of second-order nonlinear delay dynamic equations on time scales.

The study of almost automorphic functions in Bochner's sense have attracted several mathematicians since the publication of N'Guérékata's book in 2001. Recently, their applications to fractional differential equations have become an emerging field. A new and general existence and uniqueness theorem of almost automorphic solutions for the semilinear fractional differential equation

$$
D_{\alpha t} u(t)=A u(t)+D_{(\alpha-1) t} f(t, u(t)), \quad 1<\alpha<2,
$$

in complex Banach spaces, with Stepanov-like almost automorphic coefficients is obtained, and applications to fractional relaxation-oscillation equations are presented. The method used here can be applied successfully to a large class of fractional differential equations.

Another topic encountered in this issue is the existence of asymptotically almost periodic mild solutions for a class of abstract partial neutral integrodifferential equations with 
unbounded delay. The study of such equations is motivated by different concrete examples in various technical fields. For instance the equation

$$
\frac{d}{d t}\left[u(t)-\lambda Z \int_{-\infty}^{t} C(t-s) u(s) d s\right]=A u(t)+\lambda Z \int_{-\infty}^{t} B(t-s) u(s) d s-p(t)+q(t)
$$

arises in the study of the dynamics of income, employment, value of capital stock, and cumulative balance of payment.

Abstract partial neutral differential equations also appear in the theory of heat conduction. In the classic theory of heat conduction, it is assumed that the internal energy and the heat flux depend linearly on the temperature and on its gradient.

Under these conditions, the classic heat equation describes sufficiently well the evolution of the temperature in different types of materials. However, this description is not satisfactory in materials with fading memory. In the theory developed by J. Nunziato, M. E. Gurtin, and A. C. Pipkin, the internal energy and the heat flux are described as functionals of $u$ and $u_{x}$. An abstract and more general version of neutral system describing such phenomena is considered. The existence and qualitative properties of an exponentially stable resolvent operator for a class of integrodifferential system is studied.

The theory of functional differential equations has emerged as an important branch of nonlinear analysis. It is worthwhile mentioning that several important problems of the theory of ordinary and delay differential equations lead to investigations of functional differential equations of various types (see the books by Hale and Verduyn Lunel, $\mathrm{Wu}$, and articles by Liang, Xiao, Mophou, N'Guérékata, Benchohra, Lizama, Hernandez, etc. and the references therein). On the other hand, the theory of fractional differential equations is also intensively studied and finds numerous applications in describing real world problems (see e.g., the monographs of Lakshmikantham et al., Vatsala, Podlubny, and the papers of Agarwal et al., Benchohra et al.). In this issue, the existence of mild solutions to various fractional differential equations with nonlocal conditions or with infinite delay is studied using classical fixed point theorems.

Also, recently, the study of max-type difference equation attracted a considerable attention. Although max-type difference equations are relatively simple in form, it is unfortunately extremely difficult to understand thoroughly the behavior of their solutions. The max operator arises naturally in certain models in automatic control theory. Furthermore, difference equation appear naturally as a discrete analogue and as a numerical solution of differential and delay differential equations having applications in various scientific branches, such as ecology, economy, physics, technics, sociology, and biology. Asymptotic behavior of the positive solutions of a general difference equations is studied in a fine paper, improving recent results by Yang et al.

G. M. N'Guérékata

T. Diagana

A. Pankov 